\title{
Periosteum-derived micro-grafts for tissue regeneration of human maxillary bone
}

Riccardo d'Aquino ${ }^{1,2}$, Letizia Trovato ${ }^{2 *}$, Antonio Graziano ${ }^{2}$, Gabriele Ceccarelli ${ }^{3}$, Gabriella Cusella de Angelis ${ }^{3}$, Angelo Marangini ${ }^{4}$, Alessandro Nisio ${ }^{5}$, Massimo Galli ${ }^{6}$, Massimo Pasi ${ }^{7}$, Marco Finotti ${ }^{8}$, Saturnino M Lupi ${ }^{9}$, Silvana Rizzo ${ }^{9}$ and Ruggero Rodriguez Y Baena ${ }^{9}$

${ }^{1}$ Oral Surgeon - C.so Galileo Ferraris 63 Turin and Via R. G. De Ayala 9, Naples Italy

${ }^{2}$ Human Brain Wave srl, corso Galileo Ferraris 6, 10128 Turin, Italy

${ }^{3}$ Department of Public Health, Experimental Medicine and Forensics, University of Pavia, Pavia, Italy

${ }^{4}$ Oral Surgeon,Naples, Italy

${ }^{5}$ Oral Surgeon, Bari, Italy

${ }^{6}$ Oral Surgeon, Pistoia Italy

${ }^{7}$ Dentistry Service, San Raffaele Hospital, Milan, Italy

${ }^{8}$ Oral Surgeon, Padova, Italy

${ }^{9}$ School of Dentistry, Department of Clinico-Surgical, Diagnostic and Pediatric Sciences, University of Pavia, Pavia, Italy

\begin{abstract}
The bone regeneration is one of the most important challenges for regenerative medicine. In maxillo-facial area, bone resorption of the alveolar crest occurs after tooth extraction and leads to several risks for rehabilitation treatments, including dental implants procedures. Goal of our study was to demonstrate the efficacy of an innovative clinical protocol of bone tissue engineering called Rigenera protocol, aimed to create and optimize bio-complexes constituted by collagen biomaterial and human autologous periosteum-derived micro-grafts. We assessed the capacity of these bio-complexes to prevent the bone resorption in the alveolar crest with respect to simple collagen performing histological evaluations of neo-formed osseous tissue. We demonstrated that autologous bio-complexes significantly reduced the bone resorption of both horizontal and vertical dimension of alveolar crest when compared to collagen alone. We also showed that these bio-complexes accelerate the ossification process triggering the formation of new osseous tissue after 45 days from treatment and increasing the calcified matrix after 60 days and until to 120 days with respect to collagen alone. Taken together, these data showed the efficacy of bio-complexes composed by periosteum-derived micro-grafts and collagen in the alveolar ridge preservation through a reduction of bone resorption and an enhancement of new osseous tissue formation.
\end{abstract}

\section{Introduction}

The bone regeneration represents today an important challenge in the field of regenerative medicine and in craniofacial regeneration [1]. In particular, in the dental implants procedures, the placement of the implant can be limited by deficiency of the alveolar ridge caused by resorption of bone after extraction of a tooth which is followed by significant dimensional changes in the alveolar crest [2]. This leads to a reduction of the height and width of the alveolar ridge and to a reduced amount of available residual bone for implant placement. For this reason, alveolar ridge preservation (ARP) is a key procedure of contemporary clinical dentistry. Several clinical techniques and bone substitute materials can be used to fill the socket after tooth extraction. For all of them, the aim is to keep the shape and the size of the bone socket of the extracted tooth allowing the positioning of dental implants [3]. Bone regeneration is a complex process requiring the migration and proliferation of specific cells to the healing area in order to provide the biological substrate for the growth of new tissue. Although the exact mechanisms regulating the bone regeneration processes are yet to be understood, several methods for predictable bone reconstruction have been proposed [4]. Regarding alveolar socket preservation, the grafting materials commonly used include specific autogenous, allografts xenografts, or alloplasts chips [5]. The use of autologous bone is considered the gold standard in bone regeneration; however, this approach is limited due to the small amount of bone available if collected intra-orally, and by postoperative morbidity and reabsorption if collected from extra-oral sites (e.g., iliac crest, calvaria, etc.) [6-7]. Also the others grafting materials display several limits, like the absence of viable cell populations carried by allografts, xenografts, and alloplasts, which determine only osteoconductive properties [5]. To overcome these limitations, the use of growth factors incorporated in carriers, the stimulation of the selective production of growth factors by gene therapy, and the delivery of expanded cellular constructs are being used in different areas of maxillofacial reconstruction [8]. On the other hand, the use of mesenchymal stem cells (MSCs) represents a promising approach in particular for oral bone regeneration cause these cells were also identified in the tooth region, in the perivascular niche of dental pulp and periodontal ligament [9].

Periosteum is a thin fibrous layer that covers bones and provides

Correspondence to: Letizia Trovato, $\mathrm{PhD}$, Human Brain Wave srl, corso Galileo Ferraris 6, 10128 Turin, Italy, Tel: + 39011 9934508, E-mail: letizia.trovato@unito.it

Key words: autologous, bone, micro-grafts, periosteum, regeneration, stem cells

Received: February 22, 2016; Accepted: March 03, 2016; Published: March 07, 2016 
a niche for pluripotent cells and a source for molecular factors that modulate cell behavior. Periosteum-derived progenitor cells (PDPCs) are widely used in several clinical applications such as cartilage regeneration, bone healing and oral-maxillofacial tissue engineering [10].

The current study focused on the regeneration, repair and rebuilding of craniofacial tissues combining implantable biocompatible materials with micro-graft technologies based on Rigenera protocol. In particular, we demonstrated the efficacy of bio-complexes composed by periosteum-derived autologous micro-grafts and collagen with respect to collagen alone in the alveolar ridge preservation following teeth extraction.

\section{Patients and methods}

\section{Patients}

Our protocol was approved by the University of Pavia Ethical Committee (official deposit 31/03/2014) and included a total of thirtyfive patients ( 21 females and 14 males), ranging in age from 25 to 64 years and under treatment for periodontal disease, participated in this study. Eligibility criteria for participants and settings were the following: extraction needed for 2 multiple roots teeth, with closely comparable conditions and indication to undergo an implantsupported prosthesis; no systemic disease; no pregnancy (for females); no routine drug use. All patients were physically healthy, with no underlying systemic diseases as determined by medical history screening. Before entering the study, the patients were informed of the nature of the investigation, and signed an informed consent form. All patients have received basic periodontal therapy and exhibited good oral hygiene. If an abscess was present, systemic antibiotic therapy was given 2 weeks before the extraction of the scheduled tooth. The patients had one tooth scheduled for extraction as a test site and the other one as control site. In order to perform the socket preservation, in the test site we grafted bio-complexes composed by biomaterial (collagen) and periosteum-derived micro-grafts, while in the control site we used only the biomaterial (collagen).

Patients were subjected to professional oral hygiene one week before surgery. They were then instructed to perform domiciliary hygiene of the oral cavity correctly, which consisted in washing the mouth with $0.2 \%$ Chlorhexidine (CHX) after tooth brushing, twice a day until surgery was performed.

\section{Autologous micro-grafts collection by Rigenera protocol}

To disaggregate the periosteum samples, we performed a Rigenera protocol, based on the use of a disposable medical device called Rigeneracons (Human Brain Wave srl, Turin, Italy), a biological disruptor of human connective tissues able to filter and select progenitor cells with a size of 50 micron. This device allows to obtain autologous micro-grafts ready to use in a safe and easy manner without extensive manipulation. Every device owns a grid with 100 exagonal holes and any hole is embraced by six micro-blades designed for efficient cutting of hard and soft tissues. Briefly, we collected a small piece of tissue (1-2 $\mathrm{mm}$ up to $10 \mathrm{~mm}$ ) that was inserted in the Rigeneracons device with $1 \mathrm{ml}$ of physiological solution (Figure 1A and 1B). After this, we disaggregated the tissue inserting the filter in the Rigenera machine that by a rotation $(75 \mathrm{r} / \mathrm{min}$ and $15 \mathrm{Ncm})$ activates the disruption. After 2 minutes, the micro-grafts suspension was collected with a syringe using the dedicated hole (Figure 1C). The cell suspension was used to soak a collagen sponge for 10 minutes in order to build a bio-complex that was directly grafted on the alveolar socket (Figure 1D).
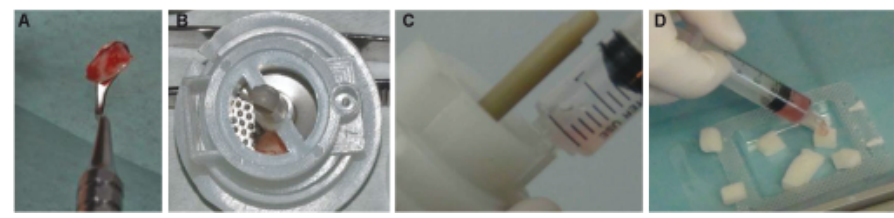

Figure 1. Schematic representation of Rigenera protocol. (A) Periosteum sample collection of size 1-2 mm up to $10 \mathrm{~mm}$; (B) Insert of periosteum sample in the Rigeneracons medical disposable where was added $1 \mathrm{ml}$ of physiological solution. The Rigeneracons was then inserted in the Rigenera machine to activate the disruption $(75 \mathrm{r} / \mathrm{min}$ and $15 \mathrm{Ncm})$. (C) Collection of cell suspension containing autologous periosteum-derived micro-grafts with a syringe using the dedicated hole. (D) Soak of collagen sponge with micro-grafts for 8-10 minutes to create a bio-complex (test site).

\section{Surgery and grafting procedure}

In the study, 8 different operators in 8 different clinical centers performed the surgeries as described. Following local anesthesia, the teeth were extracted. The sockets were thoroughly debrided to remove granulation tissue. Measurements were taken to evaluate the resorption after the healing process. Using a probe, we determined the horizontal and vertical dimension, measuring the space between the vestibular and lingual cortex and referring the cervical line of adiacent teeth, sited before and after the surgical site, respectively. A resin jig was used to record the points of observation. After tooth extraction, a small sample of periosteum was collected in the nearby site and the autologous micro-grafts obtained as above described were gently endorsed with a syringe onto a collagen sponge scaffold (Gingistat, Vebas, San Giuliano Milanese, Italy) and let for a time of 8-10 minutes before grafting. Before grafting procedure, intrardicoular bone septum was removed in order to obtain a large alveolar defect with only one cavity and afterwards the bio-complex was grafted into the site. The sponge/micro-grafts complex was used to fill the space left by the extraction procedure of test $(\mathrm{T})$ site, while a sponge without micro-grafts was used to fill the control (C) site in order to achieve natural healing by clot. The flaps in both the $\mathrm{T}$ and $\mathrm{C}$ sites were sutured, without any attempt to achieve primary closure of the surgical wound.

The patients rinsed with chlorhexidine digluconate $0.2 \%$, twice/ daily for a 2 -week period. The sutures were removed 1 week following the extraction. No antibiotic therapy was administered and only analgesic treatment was recommended in case of postoperative pain.

\section{Post-surgical evaluation of patients}

Clinical and radiological controls were performed. The first control was scheduled at day 7 after surgery, when sutures were removed and a periapical Xray was performed by means of Rinn colllimator. Edema, presence of inflammation and functionality were also clinically evaluated. The installation of the fixture at $\mathrm{T}$ and $\mathrm{C}$ sites was performed to 45 until 90 day after the extractions. Following local anesthesia and elevation of full-thickness buccal and lingual flaps, new measurements were repeated in a manner similar to that described during the baseline clinical examination. The distance between the three landmarks from the jig, and the newly located position of the alveolar crest was recorded. The holes were then enlarged and deepened to permit the insert of endosseous implants.

\section{Histological analysis}

A surgical trephine with $3 \mathrm{~mm}$ inner diameter and a minimum of $6 \mathrm{~mm}$ length was used to harvest a sample of bone from the central part of the pre-existing sockets. The holes were then refined to receive endosseus implants. All samples were collected from control and test sites (collagen alone and collagen plus micro-grafts, respectively) at 
45, 60, 90 and 120 days. The core of the bone was immediately fixed in Formalin 10\%, decalcified in EDTA solution (Osteodec, BioOptica, Mian, Italy) and paraffin-embedded. $5 \mu \mathrm{m}$-thick-sections were prepared using a microtome (Leica Biosystem, Milan, Italy) and mounted on coated glass slides. Subsequently, sections were deparaffinized in xylene, immersed in decreasing concentrations of ethanol, and rehydrated in water. Finally, sections were stained with Hematoxylin and Eosin for histological evaluation to define new periodontal formation.

\section{Statistical analysis}

The statistical analysis of post-surgical measurements was performed using two-tailed Student $t$ test considering significant a $\mathrm{P}$ value $\leq 0.05$. All analyses were performed using GraphPad 5.0 software (San Diego, CA).

\section{Results}

Our data showed the difference between the normal healing and the micro grafts application in the alveolar socket. From clinical point of view, the healing following tooth extractions occurred uneventfully in all the $\mathrm{T}$ and $\mathrm{C}$ sites. One week following the extractions, the clinical appearance of the soft tissues around the extraction sockets was similar in the $\mathrm{T}$ and $\mathrm{C}$ groups. After 2 weeks, the sockets in the $\mathrm{T}$ and $\mathrm{C}$ groups were covered by soft tissue. The post-operative period was more or less the same for both groups. Only few analgesic supports were used by 7 subjects in the first 2 days. The most interesting data was the surgical measurements taken at the time of second stage surgery that revealed an overall significant lesser resorption of the alveolar crest in the Test group (T) compared to Control group (C). In fact, the horizontal resorption in the $\mathrm{T}$ sites was $38.3 \%$ lesser than in $\mathrm{C}$ sites (Figure $2 \mathrm{~A}$ ), while the vertical one was in $\mathrm{T}$ sites $36.5 \%$ lesser than $\mathrm{C}$ sites (Figure 2B).

We further evaluated the histological sections of samples collected by trephine as above described after 45, 60, 90, 120 days from treatment. The comparison of the samples treated with collagen alone and periosteum-derived micro-grafts with collagen showed that the ossification process was much faster in the Test group than Control group at each point. In particular, at 45 days, the Control group is characterized by the presence of inflammatory cells and no bone formation appeared. On the contrary, Test group exhibited bone formation already at 45 days, with an increase of calcified matrix at 60, 90 and 120 days with respect to Control group where the organic matrix is more evident (Figure 3).
A

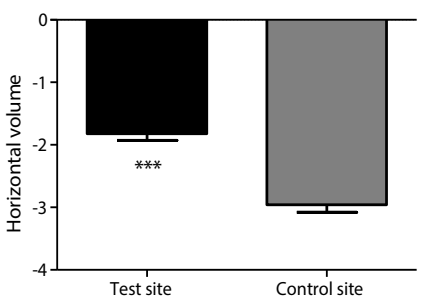

B

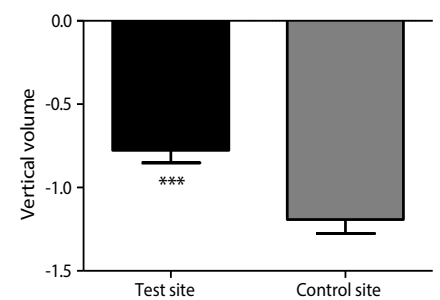

Figure 2. Surgical measurements of horizontal (A) and vertical dimension (B) of alveolar crest in the Test site and Control site during sample collection. The horizontal and vertical dimension were determined measuring the space beetwen the vestibular and lingual cortex and referring the cervical line of adiacent teeth, sited before and after the surgical site, respectively. A resin jig was used to record the points of observation. The figure shows a significant reduction of bone resorption in the test site (micro-grafts with collagen) with respect to control site (collagen alone) $(* * * \mathrm{P} \leq 0.001$ test site vs. control site, calculated by $\mathrm{T}$ test analysis using Graphpad 5.0 software).

\section{Collagen plus micrografts (test) Collagen alone (control)}
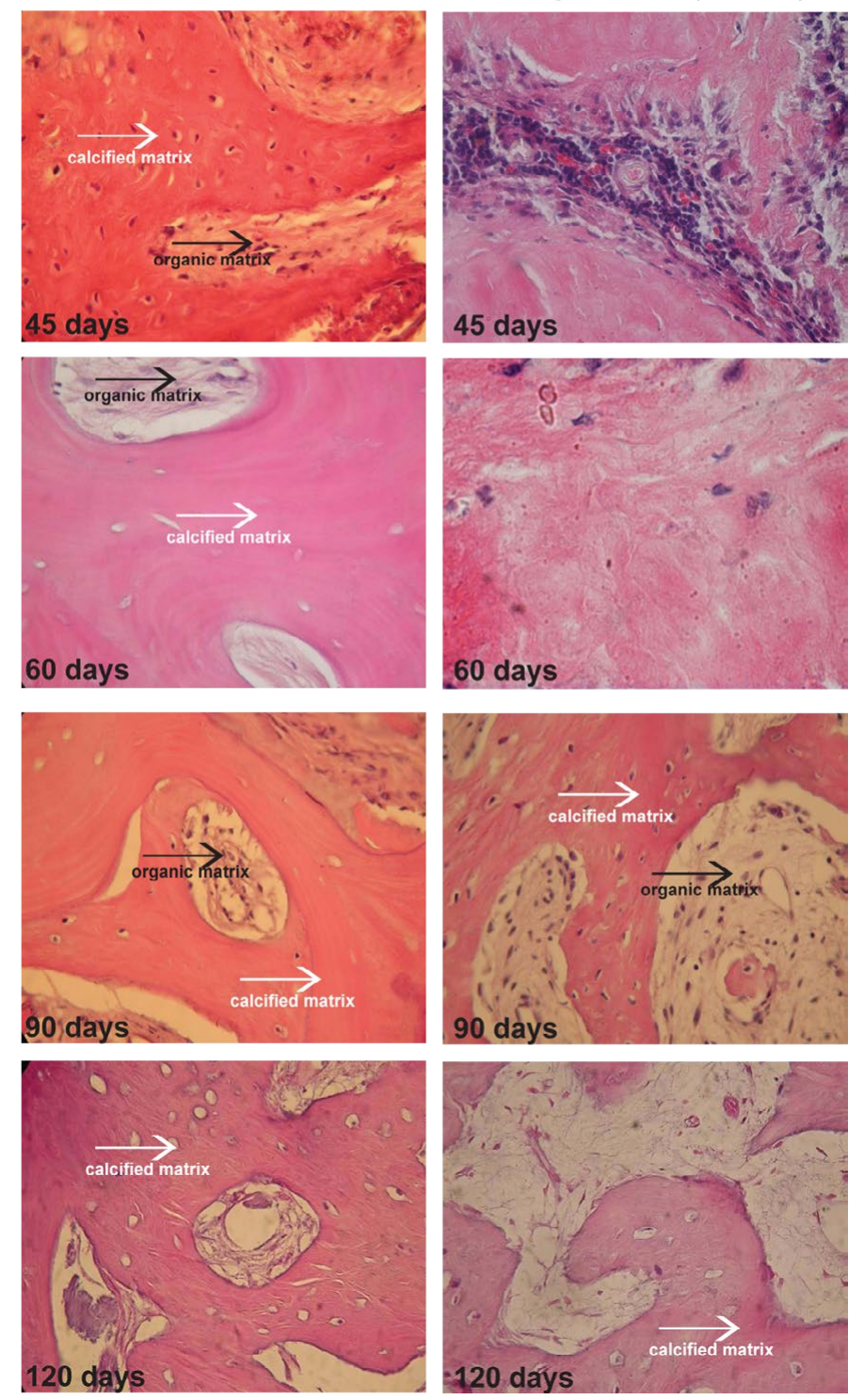

Figure 3. Histological evaluation of core bone sections collected from Test and Control sites after 45, 60, 90 and 120 days from treatment. The sections were stained with Hematoxylin and Eosin as indicated in the appropriate section of patients and methods. The white arrows indicate the calcified matrix, while the black arrows indicate the organic matrix. The comparison of the samples treated with collagen alone (control) and collagen with periosteum-derived micro-grafts (test) showed that the ossification process is much faster in the Test group than Control group at each point.

\section{Discussion}

The alveolar socket or ridge preservation is defined as the procedure of arresting or minimizing the alveolar ridge resorption following tooth extraction for future treatments purposes, including placement of dental implants, but there is a need for of its effectiveness. A recent review of the literature [5] reported limited clinical evidences that socket preservation can reduce bone loss compared to the simple tooth extraction without preserving treatments for dental implant placement purposes and also no convincing evidence of any significant difference between different materials and barriers used for socket preservation [5]. It should be underlined there are several ARP techniques, including 
the placement of different grafting materials with or without the use of membranes, to preserve and minimize bone resorption [11-12]. In this study we showed that autologous periosteum-derived micrografts with collagen was more effective to preserve the horizontal and vertical dimension with respect to collagen alone and we challenged the obtained bone regeneration with histological analysis. The efficacy of micro-grafts was already showed in the dentistry field where it has been reported an enhancement of periodontal tissue regeneration [13] and bone regeneration of atrophic maxilla [14]. It has been also reported that dental pulp-derived micro-grafts are effective to restore human mandible bone defects [15] and to promote the closure of noncontained intrabony defects in chronic periodontitis [16].

The efficacy of autologous micro-grafts was also reported for other clinical applications such as wound healing of postoperative complex wounds [17], post traumatic lesions [18] and pathological scars [19]. The autologous grafts are surely preferable although they exhibit some limits in terms of tissue sacrifice and morbidity for the donor site [67]. In addition, a crucial issue for the auto-grafts remains the cell and tissue viability that after collection dramatically decreases, reducing the auto-graft regenerative capacities [6-7]. The cells death is due to the vessel interruption and the subsequent reduction of the feeding for the tissue, but also reducing the dimensions of the grafts, fragmenting the bone tissues to small particles or chips to allow a greater contact between bone chips and biological fluids, cell death problem remains.

In this study we obtained the autologous micro-grafts from a sample of periosteum which was disaggregated by Rigenera protocol, and we previously demonstrated that these micro-grafts maintain both an high cell viability and high positivity to stem cell markers such as CD7, CD90, CD105 [20]. Furthermore, it has been also reported that human periosteal cells maintain the properties to self-commitment towards osteogenesis representing an innovative stem cells source for maxillo-facial tissue engineering applications [21].

The only way to use the regenerative potential of the tissue up to now in clinical medicine was in the grafting procedures, moving matrix, cells and factors from a healthy niche to an injured niche. The Rigenera protocol is a novel approach to the clinical regeneration of the human tissues through autologous micro-graft obtained by a CE Class I innovative medical devices called Rigeneracons. The goal of this protocol is to help the collection of viable progenitor after a specific filtration in a sterile suspension that at the end of the process is ready to use as itself or after a mixing with a biomaterial to perform a graft of a bio-complex, as already reported [18].

The quality and quantity of the available bone for implant is fundamental in terms of both predictability of the implant survival and the maintenance of its long-term stability. In the case of alveolar ridges with insufficient bone volume or unfavorable vertical, horizontal or sagittal intermaxillary relationships, additional surgical procedures can be necessary to reconstruct and augment the deficiency [22]. For this reason, alveolar ridge has become widely used in the augmentation of bone allowing to minimize the amount of horizontal and vertical volume lost and to maximize formation of new bone after removal of a tooth [23]. This approach is quite technically sensitive, nevertheless it requires particular surgical skills, and it is expensive for the patient and potentially dangerous for the risk of wound infection and rejection. Cardaropoli et al. (2012) reported that filling the socket with a bone graft was an effective way to reduce resorption of the alveolar ridge, because the bone grafts maintained the vertical and horizontal dimensions of the ridge [24]. On the other hand, in this study we showed that micro- grafts with collagen application significantly prevented the reduction of vertical and horizontal dimensions of the ridge with respect to collagen alone. Furthermore, histological analyses demonstrated that the combination of micro-grafts with collagen accelerated ossification processes in test group with respect to control group already at 45 days with an increase of calcified matrix. Hence, Rigenera protocol facilitates and accelerates the formation of new osseous tissue.

In conclusion, our data reported the efficacy of autologous micro-grafts derived from periosteum samples in reducing the bone resorption of alveolar crest suggesting that this can be represent an optimal approach of alveolar ridge preservation.

\section{Conflict of interest}

The authors d'Aquino R, Trovato L and Graziano A are components of Medical and Scientific Division of Human Brain Wave, the company that has developed the Rigenera protocol and Rigeneracons disposable device.

\section{References}

1. Pagni G, Kaigler D, Rasperini G, Avila-Ortiz G, Bartel R, et al. (2012) Bone repair cells for craniofacial regeneration. Adv Drug Deliv Rev 64: 1310-1319. [Crossref]

2. Avila-Ortiz G, Elangovan S, Kramer KW, Blanchette D, Dawson DV (2014) Effect of alveolar ridge preservation after tooth extraction: a systematic review and metaanalysis. J Dent Res 93: 950-958. [Crossref]

3. Darby I, Chen ST, Buser D (2009) Ridge preservation techniques for implant therapy Int J Oral Maxillofac Implants 24 Suppl: 260-271. [Crossref]

4. Dimitriou R, Jones E, McGonagle D, Giannoudis PV (2011) Bone regeneration: current concepts and future directions. BMC Med 9: 66. [Crossref]

5. Atieh MA, Alsabeeha NH, Payne AG, Duncan W, Faggion CM, et al. (2015) Interventions for replacing missing teeth: alveolar ridge preservation techniques for dental implant site development. Cochrane Database Syst Rev 5: CD010176. [Crossref]

6. Nkenke E, Weisbach V, Winckler E, Kessler P, Schultze-Mosgau S, et al. (2004) Morbidity of harvesting of bone grafts from the iliac crest for preprosthetic augmentation procedures: a prospective study. Int J Oral Maxillofac Surg 33: 157-163. [Crossref]

7. Barone A, Ricci M, Mangano F, Covani U (2011) Morbidity associated with iliac crest harvesting in the treatment of maxillary and mandibular atrophies: a 10 -year analysis. $J$ Oral Maxillofac Surg 69: 2298-2304. [Crossref]

8. Padial-Molina M, Rios HF (2014) Stem cells, scaffolds and gene therapy for periodontal engineering. Current Oral Health Reports 1: 16-25

9. Shi S, Bartold PM, Miura M, Seo BM, Robey PG, et al. (2005) The efficacy of mesenchymal stem cells to regenerate and repair dental structures. Orthod Craniofac Res 8: 191-199. [Crossref]

10. Ferretti C, Mattioli-Belmonte M (2014) Periosteum derived stem cells for regenerative medicine proposals: Boosting current knowledge. World J Stem Cells 6: 266-277. [Crossref]

11. Hämmerle CH, Araújo MG, Simion M; Osteology Consensus Group 2011 (2012) Evidence-based knowledge on the biology and treatment of extraction sockets. Clin Oral Implants Res 23 Suppl 5: 80-82. [Crossref]

12. Vignoletti F, Matesanz P, Rodrigo D, Figuero E, Martin C, et al. (2012) Surgical protocols for ridge preservation after tooth extraction. A systematic review. Clin Oral Implants Res 23 Suppl 5: 22-38. [Crossref]

13. Graziano A, Carinci F, Scolaro S, D’Aquino R (2013) Periodontal tissue generation using autologous dental ligament micro-grafts: case report with 6 months follow-up. AOMFS 1: 20.

14. Brunelli G, Motroni A, Graziano A, D'Aquino R, Zollino I, et al. (2013) Sinus lift tissue engineering using autologous pulp micro-grafts: A case report of bone density evaluation. J Indian Soc Periodontol 17: 644-647. [Crossref]

15. d'Aquino R, De Rosa A, Lanza V, Tirino V, Laino L, et al. (2009) Human mandible bone defect repair by the grafting of dental pulp stem/progenitor cells and collagen sponge biocomplexes. Eur Cell Mater 18: 75-83. [Crossref]

16. Aimetti M, Ferrarotti F, Mariani GM, Cricenti L, Romano F (2013) Use of Dental Pulp 
Stem Cells/Collagen Sponge Biocomplex in the Treatment of Non-contained Intrabony Defects: A Case Series. Clinic Adv in Periodontics 5: 2.

17. Giaccone M, Brunetti M, Camandona M, Trovato L, Graziano A (2014) A New Medical Device, Based on Rigenera Protocol, in the Management of Complex Wounds. J Stem Cells Res Rev \& Rep 1: 3 .

18. Purpura V, Bondioli E, Graziano A, Trovato L, Melandri D, et al. (2014) Tissue Characterization After a New Disaggregation Method for Skin Micro-Grafts Generation. J Vis Exp e53579.

19. Svolacchia F, De Francesco F, Trovato L, Graziano A, Ferraro GA (2016) An innovative regenerative treatment of scars with dermal micrografts. J Cosmet Dermatol [Crossref]

20. Trovato L, Monti M, Del Fante C, Cervio M, Lampinen M, et al. (2015) A New
Medical device rigeneracons allows to obtain viable micro-grafts from mechanical disaggregation of human tissues. J Cell Physiol 230: 2299-2303. [Crossref]

21. Ceccarelli G, Graziano A, Benedetti L, et al. (2016) Osteogenic Potential of Human Oral-Periosteal Cells (PCs) Isolated From Different Oral Origin: An In Vitro Study. $J$ Cell Physiol 231: 607-612. [Crossref]

22. Aghaloo TL, Moy PK (2007) Which hard tissue augmentation techniques are the most successful in furnishing bony support for implant placement? Int J Oral Maxillofac Implants 22 Suppl: 49-70. [Crossref]

23. Wang RE, Lang NP (2012) Ridge preservation after tooth extraction. Clin Oral Implants Res 23 Suppl 6: 147-156. [Crossref]

24. Cardaropoli D, Tamagnone L, Roffredo A, Gaveglio L, Cardaropoli G (2012) Socket preservation using bovine bone mineral and collagen membrane: a randomized controlled clinical trial with histologic analysis. Int J Periodontics Restorative Dent 32: 421-430. [Crossref]

Copyright: $(02016$ d'Aquino R. This is an open-access article distributed under the terms of the Creative Commons Attribution License, which permits unrestricted use, distribution, and reproduction in any medium, provided the original author and source are credited. 